\title{
A semi-classical study of the Casati-Prosen triangle map
}

\author{
M Degli Esposti† S O’Keefe $\ddagger$ and B Winn $\dagger$ \\ $\dagger$ Dipartimento di Matematica, Università di Bologna, 40127 Bologna, Italy. \\ $\ddagger$ School of Mathematics, University of Bristol, Bristol BS8 1TW, UK. \\ E-mail: desposti@dm.unibo.it, stephen.okeefe@bristol.ac.uk, winn@dm.unibo.it
}

\begin{abstract}
We investigate the semi-classical properties of a two-parameter family of piece-wise linear maps on the torus known as the Casati-Prosen or triangle map. This map is weakly chaotic and has zero Lyapunov exponent. A correspondence between classical and quantum observables is established, leading to an appropriate statement regarding equidistribution of eigenfunctions in the semi-classical limit. We then give a full description of our numerical study of the eigenvalues and eigenvectors of this family of maps. For generic choices of parameters the spectral and eigenfunction statistics are seen to follow the predictions of the random matrix theory conjecture.
\end{abstract}

PACS number: $05.45 . \mathrm{Mt}$

AMS classification scheme numbers: 81Q50, 81S05

Submitted to: Nonlinearity

\section{Introduction and notations}

Quantum chaology is concerned with the semi-classical study of systems whose classical counterparts are chaotic. This study has focused on the behaviour of energy levels and corresponding eigenfunctions in the classical limit. It turns out that the semi-classical behaviour of these quantities is markedly different if the system is chaotic, compared with an integrable system. For the spectrum of energy levels two important conjectures are the Bohigas-GiannoniSchmit conjecture [12] that classically chaotic systems exhibit correlations in the quantum spectrum like those in eigenvalues of random matrices; and the conjecture [7] of Berry and Tabor, that for classically integrable systems the energy levels are completely uncorrelated. For eigenfunctions one has the semi-classical eigenfunction hypothesis [6, 44]. Put simply this asserts that a particular phase-space representation of the energy eigenfunctions, (the Wigner distribution) semi-classically converges to the Dirac delta distribution supported on the region of phase space explored by typical trajectories in long times. For a chaotic (so in particular, ergodic) system, this set is the whole surface of constant energy. For an integrable system, motion is confined to tori in phase space, so the semi-classical eigenfunction hypothesis in this case implies that the Wigner function condenses onto these tori in the classical limit.

A wide variety of chaotic systems have been investigated, both numerically and analytically, in the intervening 20-or-so years, and we are now convinced that these conjectures give an accurate picture of the behaviour of generic systems.

Nevertheless, the term "chaotic" contains a whole hierarchy of behaviours such as: ergodicity, mixing, hyperbolicity, et cetera, and it is of interest to know which of these properties are necessary 
to recover generic effects. As an example we mention the skew translations. These are ergodic maps of the torus, which possess no stronger chaotic properties. They are not strongly or weakly mixing. In [40] it was proved that when quantised all eigenfunctions of the corresponding quantum map equidistribute in the classical limit. The spectral statistics, however, fail to obey the predictions of random matrix theory [2]. Most systems which are believed to behave generically with respect to the Bohigas-Giannoni-Schmit conjecture have quite strong chaotic properties. The original study in [12] was of the quantised Sinai billiard which is a $K$-system. As an example of quantum maps with random matrix statistics we mention the family of perturbed cat maps [4] which are Anosov systems, so strongly mixing, with exponential proliferation of periodic orbits and positive entropy.

In the present work we present a family of maps with much weaker chaotic behaviour, which nevertheless for certain choices of the governing parameters seem to behave generically with respect to the Bohigas-Giannoni-Schmit conjecture. The map is conjectured to be ergodic and strongly mixing, but with a sub-exponential decay of correlations, and zero Lyapunov exponent. There are no periodic orbits. We believe this to be currently the weakest chaotic map for which random matrix statistics are observed in the quantum spectrum. For other choices of parameters the chaotic properties are reduced further and we observe a class of intermediate statistics which have also been observed for other weakly chaotic maps. In all cases we have equidistribution of a density one subset of eigenfunctions, provided only that the parameters are chosen such that the map is ergodic.

\subsection{The Casati-Prosen triangle map}

We work on the torus $\mathbb{T}:=\mathbb{R} / \mathbb{Z}$. We define for $\alpha, \beta \in \mathbb{R}$ the family of area-preserving torus maps $F_{\alpha, \beta}: \mathbb{T}^{2} \rightarrow \mathbb{T}^{2}$ by

$$
F_{\alpha, \beta}\left(\begin{array}{c}
q \\
p
\end{array}\right):=\left(\begin{array}{c}
q+2(p+\beta+\alpha \theta(q)) \\
p+\beta+\alpha \theta(q)
\end{array}\right) \bmod 1
$$

where $\theta: \mathbb{T} \rightarrow\{-1,1\}$ is defined by

$$
\theta(q):=\left\{\begin{aligned}
1, & 0 \leqslant q<\frac{1}{2} \\
-1, & \frac{1}{2} \leqslant q<1 .
\end{aligned}\right.
$$

This map coincides with the map introduced in [17] but for the factor of 2 which appears. This factor simplifies the quantisation procedure without fundamentally altering the dynamical properties of the map. This is a parabolic, piece-wise linear map with discontinuities at the lines $q=0$ and $q=1 / 2$. The map is related to the Poincaré map of a particle inside a triangular billiard with one very small angle, and for this reason is often referred to as the triangle map.

We observe that $F_{\alpha, \beta}$ can be written as a composition of three simpler maps,

$$
F_{\alpha, \beta}=B \circ R_{\beta} \circ G_{\alpha}
$$

where the constituent maps are defined by:

$$
\begin{aligned}
B\left(\begin{array}{l}
q \\
p
\end{array}\right):=\left(\begin{array}{ll}
1 & 2 \\
0 & 1
\end{array}\right)\left(\begin{array}{l}
q \\
p
\end{array}\right) \bmod 1, \\
R_{\beta}\left(\begin{array}{c}
q \\
p
\end{array}\right):=\left(\begin{array}{c}
q \\
p+\beta
\end{array}\right) \bmod 1, \\
G_{\alpha}\left(\begin{array}{l}
q \\
p
\end{array}\right):=\left(\begin{array}{c}
q \\
p+\alpha \theta(q)
\end{array}\right) \bmod 1 .
\end{aligned}
$$

In fact $R_{\beta}$ and $G_{\alpha}$ commute because they only act in the $p$ direction. $G_{\alpha}$ is the discontinuous part of the map. It cuts the torus along the lines $q=0$ and $q=1 / 2$, and translates the two pieces in opposite directions parallel to the cuts. The map $R_{\beta}$ is simply a translation in the $p$ direction, and $B$ is a parabolic skew translation, which causes a linear stretching. The overall map is marginally 

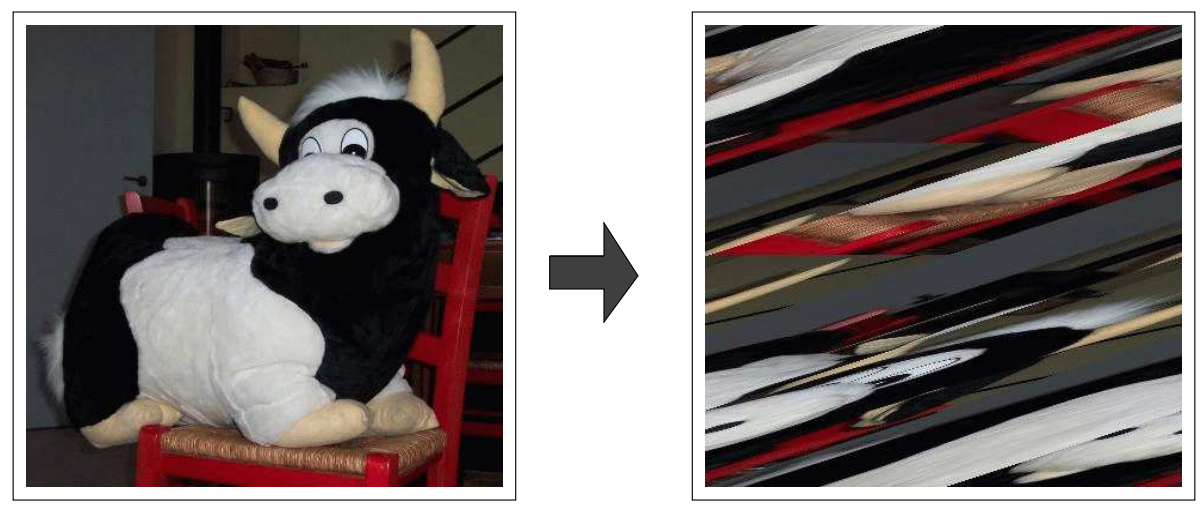

Figure 1. A single iteration of the triangle map.

stable; points that are initially close and separated by a non-zero vertical distance separate linearly until one of them reaches a discontinuity (see figure 1).

When $\alpha=0$ the discontinuous part of the dynamics is switched off and the map is a continuous skew translation. In this case if $\beta$ is irrational then the map is uniquely ergodic [15, 28], and there are no other chaotic features. All interesting behaviour in the triangle map for $\alpha \neq 0$ can then be traced back to the action of cutting the torus.

There are surprisingly few rigorous results about ergodic or topological properties of the map when $\alpha \neq 0$. We rely on numerical experiments and conjectures in this discontinuous case. If $\alpha$ and $\beta$ are both non-zero, and independently irrational, the motion is numerically conjectured to be ergodic and mixing [17]. The correlations of a typical classical function decay according to a power law, $\langle f(t) f(0)\rangle \propto t^{-\gamma}$, with exponent $\gamma \approx 3 / 2$. The spectral density, the inverse Fourier transform of the correlation function, is a continuous but not continuously differentiable function. In this regime, the map has no periodic orbits.

The map in this regime also exhibits exponential decay of Poincaré recurrences. This property is usually associated with hyperbolic maps, and its existence for a parabolic map marks out the triangle map for study. This phenomenon is conjectured to be related to the absence of periodic orbits [17]. Recently it has been found, numerically and supported by heuristic arguments, that the triangle map admits the universal decay of temporal correlations associated with systems lacking exponential sensitivity to initial conditions [19].

For $\alpha \neq 0$ and $\beta=0$, there are two distinct cases. If $\alpha$ is rational, the motion is pseudointegrable. For irrational $\alpha$, the motion is weakly ergodic, i.e. the rate of ergodicity is slow compared to the $\beta \neq 0$ regime. For example, the number of different values of the momentum coordinate $p_{n}$ taken by an orbit up to time $T$, with $0 \leqslant n<T$ and $n \in \mathbb{Z}$, grows at the same rate as $\log T$. We again have a power law decay of correlations, with $\gamma$ small, numerically $\gamma \approx 0.1$. It is conjectured [17] that the true value is $\gamma=0$, so that there is no decay of correlations - that is that the mixing property does not hold in this regime, but it has not yet been fully rejected by numerical experiment.

We are mainly interested in the cases where $\alpha$ is irrational, and the two regimes; $\beta$ irrational and independent of $\alpha$, and $\beta=0$. We can further classify the motion in these regimes by considering the walk determined by the function $\theta\left(q_{n}\right)$ for $n \in \mathbb{N}$. We define the walk as follows. Let $\phi_{\alpha, \beta}$ be defined as the sequence

$$
\phi_{\alpha, \beta}\left(\begin{array}{c}
q_{0} \\
p_{0}
\end{array}\right):=\left(\theta\left(q_{0}\right), \ldots, \theta\left(q_{n}\right), \ldots\right)
$$


where $\left(q_{n}, p_{n}\right)$ are the $n^{\text {th }}$ iterates of the initial point $\left(q_{0}, p_{0}\right)$ under $F_{\alpha, \beta}$. For $\beta \neq 0$, the walk $\phi_{\alpha, \beta}$ has a diffusion growing like $\sqrt{T}$ after $T$ steps, like a classical random walk with independent identically distributed steps sizes. For $\beta=0$, one observes a strongly dependent walk, characterised by logarithmic diffusion [21]. These observations have been made from numerical simulations of the walk.

Given a map on the torus, now considered as a (compact) phase space, one looks for a quantum version, a quantum map. There is no mechanical procedure for constructing a quantum map from a given classical map. In practice one needs to treat each case individually, motivated by the correspondence principle, that in the classical limit quantum and classical evolution should commute. Nevertheless, such quantum maps have proved to be a useful testing ground for the conjectures of quantum chaos [23]. We describe a quantisation procedure for the Casati-Prosen map in section 2, here mentioning only the important fact that for any map the compact phase space implies that Planck's constant can only take values that are the of the form $\hbar=1 / 2 \pi N$ for $N \in \mathbb{N}$.

Just about the only rigorously-known and universal result in quantum chaology is the Schnirelman theorem, or quantum ergodicity. This result can be traced back to [43] where it was stated without proof, that almost all eigenfunctions of a system whose classical counterpart is ergodic equidistribute over the energy shell in phase space in the classical limit. This allows for a density zero set of eigenfunctions which fail to equidistribute, and, for example, localise around periodic orbits of the system. This conjecture has been proved for Hamiltonian flows $[43,45,16,34,29,48]$ and for maps [13, 46, 47, 24], where one gets in fact equidistribution of a density one subset of eigenfunctions over the whole torus. For smooth systems the proof is robust in the sense that it requires no detailed information about either the eigenfunctions, or the dynamical properties of the system; only a correspondence principle between classical and quantum evolution, or Egorov theorem, and ergodicity of the dynamics. For discontinuous systems there can be diffraction effects, and one additionally needs good control over any discontinuities of the map. This point is discussed more fully in subsection 2.2. For maps with sharply divided phase space into ergodic and non-ergodic components an analogue of the Schnirelman theorem has been proved [39] where a subset eigenfunctions localise on the ergodic component and almost all members of the subset equidistribute over this region. It is also known that the Schnirelman theorem implies a weakened version of the semi-classical eigenfunction hypothesis for ergodic systems, provided one restricts to the subsequence of density one [3].

Regarding the eigenvalues of the quantum triangle map, we are guided by the Bohigas-GiannoniSchmit conjecture [12]. This asserts that for a generic chaotic map, the eigenvalue distributions of the corresponding quantum map will follow those of a random matrix ensemble with the same symmetry properties as the map (see [32] for a discussion of the relevant symmetries). Known violations of the Bohigas-Giannoni-Schmit conjecture for quantum maps include the unperturbed cat maps [36] and the skew translations [2]. In these cases, deviations from random matrix theory are entirely due to strong number-theoretical properties of the quantum maps.

A study [30] has recently been made of the quantised skew translations with a slightly more general quantisation scheme to that in $[40,2]$. This is particularly relevant because, as we recall, the skew translations are equivalent to the triangle map with $\alpha=0$. The results of [30] are dependent on the number-theoretical properties of the parameter $\beta$. For rational choices of $\beta$ the spectral statistics were found to be of intermediate type. For $\beta$ irrational, statistics similar to those of random matrices were observed for certain choices of Planck's constant, but these statistics do not appear to be stable, and it is not possible to say that these statistics are limiting as $\hbar \rightarrow 0$.

We now state our main results and findings to be presented in the following pages. In section 2 we introduce a method of quantising the triangle map as a map of the torus, and prove a correspondence principle (or Egorov theorem) between the classical and quantum observables. This enables us to state the appropriate Schnirelmann theorem for this map. In section 3 we review the symmetries of the classical and quantum map, which determine the eigenvalue distributions. This discussion also allows us to generate real eigenvectors for which we can define nodal domains in 
analogy with those for billiards [8]. Sections 4 and 5 give an account of our numerical investigation of the eigenvalues and eigenvectors respectively of the quantum triangle map. We find for $\alpha$ and $\beta$ irrational and linearly independent that the map behaves very much like a generic random matrix, both with respect to the eigenvalue statistics, and, for suitable choices of $\alpha$ and $\beta$ some eigenvector statistics. For $\alpha$ irrational and $\beta=0$ the map displays intermediate spectral statistics, and the nodal domain distribution also deviates from the random matrix theory prediction, although the value distributions of eigenvectors are indistinguishable between the two regimes.

Throughout this paper we make use of standard asymptotic notation $\mathrm{O}_{a}(f(N))$ which represents some quantity bounded by a constant multiple of $f(N)$ for sufficiently large $N$ (and the constant may depend on the parameter $a)$. We also define $e(x):=\mathrm{e}^{2 \pi \mathrm{i} x}$ and $e_{N}(x):=e(x / N)$. Other unfamiliar notations and concepts will be defined as and when they arise.

\section{The quantum triangle map}

The quantisation of an area-preserving map on the torus $\mathbb{T}^{2}$ is a unitary operator $U$ acting on an $N$-dimensional Hilbert space $\mathcal{H}_{N}$, where $N=(2 \pi \hbar)^{-1}$. That $N$ be an integer imposes a restriction on allowed values of $\hbar$, and the classical limit is re-cast as the limit $N \rightarrow \infty$. Historically the first torus quantisation was given in [33]. For further details we refer the reader to [23] and references therein. (See also [20] for a pedagogical introduction to the subject.)

A quantisation recipe also needs to associate to classical observables $f \in C^{\infty}\left(\mathbb{T}^{2}\right)$ a corresponding quantum observable $\hat{f}$ which is a Hermitian operator on $\mathcal{H}_{N}$. We use a variant of the Weyl quantisation although other schemes are available. The observable to be quantised $f$ is expanded as a Fourier series

$$
f(q, p)=\sum_{\mathbf{n} \in \mathbb{Z}^{2}} a_{\mathbf{n}} e\left(n_{1} q+n_{2} p\right)
$$

and $\hat{f}$ is then defined by

$$
\hat{f}:=\sum_{\mathbf{n} \in \mathbb{Z}^{2}} a_{\mathbf{n}} T(\mathbf{n}),
$$

where $T(\mathbf{n})$ are the quantised characters defined in the following way. We denote the canonical orthonormal basis of $\mathcal{H}_{N}$ by $\{|\ell\rangle: \ell=0, \ldots, N-1\}$, and occasionally use the convention that

$$
|m N+\ell\rangle=|\ell\rangle, \quad \text { for } m \in \mathbb{Z} \text {. }
$$

Then

$$
T(\mathbf{n}):=e_{N}\left(\frac{n_{1} n_{2}}{2}\right) t_{2}^{n_{2}} t_{1}^{n_{1}}
$$

where $t_{1}$ and $t_{2}$ act by

$$
t_{1}|\ell\rangle=e_{N}(\ell)|\ell\rangle
$$

and

$$
t_{2}|\ell\rangle=|\ell+1\rangle \text {. }
$$

We briefly recall a few facts about these operators. The canonical commutation relations become

$$
t_{1} t_{2}=e_{N}(1) t_{2} t_{1}
$$

which gives the relationship

$$
T(\mathbf{m}) T(\mathbf{n})=e_{N}\left(\frac{m_{1} n_{1}-n_{2} m_{2}}{2}\right) T(\mathbf{m}+\mathbf{n}) .
$$


The $N \times N$ Fourier transform matrices $\mathcal{F}_{N}$ are defined by the matrix elements

$$
\left(\mathcal{F}_{N}\right)_{j k}:=\frac{1}{\sqrt{N}} e_{N}(-j k)
$$

Then we have the following intertwinings between $t_{1}$ and $t_{2}$,

$$
\begin{aligned}
& \mathcal{F}_{N} t_{1} \mathcal{F}_{N}^{-1}=t_{2} \\
& \mathcal{F}_{N} t_{2} \mathcal{F}_{N}^{-1}=t_{1}^{-1} .
\end{aligned}
$$

Under the Weyl quantisation the commutator of two quantised observables $\hat{f}$ and $\hat{g}$ respects the Poisson bracket of $f$ and $g$ in the classical limit,

$$
\left\|\frac{2 \pi N}{\mathrm{i}}[\hat{f}, \hat{g}]-\widehat{\{f, g\}}\right\|=\mathrm{O}_{f, g}\left(N^{-1}\right) \quad \text { as } N \rightarrow \infty .
$$

This is a kinematic version of the correspondence principle. Analogously we present a quantisation of $F_{\alpha, \beta}$ which respects evolution of the map in the classical limit (see theorem 2.4 below).

\subsection{Quantising B}

We define the quantisation of $B$ by

$$
\hat{B}:=\mathcal{F}_{N}^{-1} D \mathcal{F}_{N}
$$

where $D$ is the diagonal matrix with entries

$$
D_{j k}=e_{N}\left(k^{2}\right) \delta_{j k}
$$

In general a quantisation $\hat{A}$ of a map $A$ is said to satisfy an Egorov estimate if the following is true for some class of observables $f$,

$$
\lim _{N \rightarrow \infty}\left\|\hat{A}^{-1} \hat{f} \hat{A}-\widehat{f \circ A}\right\|=0 .
$$

The Egorov estimate is the dynamical analogue of (2.15). In fact, for the map $B$, Egorov is exact for all $N$ with the quantisation (2.16) as demonstrated in the following lemma (see [40]).

Lemma 2.1. Let $f \in C^{\infty}\left(\mathbb{T}^{2}\right)$. Then

$$
\hat{B}^{-1} \hat{f} \hat{B}=\widehat{f \circ B} .
$$

Proof. Note that $\hat{B}^{-1}=\mathcal{F}_{N}^{-1} D^{-1} \mathcal{F}_{N}$, so

$$
\hat{B}^{-1} \hat{f} \hat{B}=\mathcal{F}_{N}^{-1} D^{-1} \mathcal{F}_{N} \hat{f} \mathcal{F}_{N}^{-1} D \mathcal{F}_{N} .
$$

By (2.8) it suffices to consider for $\mathbf{n} \in \mathbb{Z}^{2}$,

$$
\begin{aligned}
\mathcal{F}_{N} T(\mathbf{n}) \mathcal{F}_{N}^{-1} & =e_{N}\left(\frac{n_{1} n_{2}}{2}\right) \mathcal{F}_{N} t_{2}^{n_{2}} \mathcal{F}_{N}^{-1} \mathcal{F}_{N} t_{1}^{n_{1}} \mathcal{F}_{N}^{-1} \\
& =e_{N}\left(\frac{n_{1} n_{2}}{2}\right) t_{1}^{-n_{2}} t_{2}^{n_{1}} \quad \text { using }(2.13) \text { and }(2.14) \\
& =e_{N}\left(-\frac{n_{1} n_{2}}{2}\right) t_{2}^{n_{1}} t_{1}^{-n_{2}} \\
& =T\left(-n_{2}, n_{1}\right)
\end{aligned}
$$

But now

$$
\begin{aligned}
\left(D^{-1} \mathcal{F}_{N} T(\mathbf{n}) \mathcal{F}_{N}^{-1} D\right)|k\rangle & =e_{N}\left(\frac{n_{1} n_{2}}{2}\right) D^{-1} t_{1}^{-n_{2}} t_{2}^{n_{1}} D|k\rangle \quad \text { using }(2.20) \\
& =e_{N}\left(\frac{n_{1} n_{2}}{2}+k^{2}-n_{2}\left(k+n_{1}\right)-\left(k+n_{1}\right)^{2}\right)\left|k+n_{1}\right\rangle
\end{aligned}
$$




$$
\begin{aligned}
& =e_{N}\left(-\frac{n_{1}\left(2 n_{1}+n_{2}\right)}{2}\right) e_{N}\left(-\left(2 n_{1}+n_{2}\right) k\right)\left|k+n_{1}\right\rangle \\
& =e_{N}\left(-\frac{n_{1}\left(2 n_{1}+n_{2}\right)}{2}\right) t_{2}^{n_{1}} t_{1}^{-2 n_{1}-n_{2}}|k\rangle \\
& =T\left(-2 n_{1}-n_{2}, n_{1}\right)|k\rangle \\
& =\mathcal{F}_{N} T\left(n_{1}, n_{2}+2 n_{1}\right) \mathcal{F}_{N}^{-1}|k\rangle,
\end{aligned}
$$

giving

$$
\hat{B}^{-1} T(\mathbf{n}) \hat{B}=T\left(n_{1}, n_{2}+2 n_{1}\right) .
$$

Finally

$$
\begin{aligned}
\hat{B}^{-1} \hat{f} \hat{B} & =\sum_{\mathbf{n} \in \mathbb{Z}^{2}} a_{\mathbf{n}} \hat{B}^{-1} T(\mathbf{n}) \hat{B} \\
& =\sum_{\mathbf{n} \in \mathbb{Z}^{2}} a_{\mathbf{n}} T\left(n_{1}, n_{2}+2 n_{1}\right) \\
& =\sum_{\mathbf{n} \in \mathbb{Z}^{2}} a_{n_{1}, n_{2}-2 n_{1}} T(\mathbf{n})
\end{aligned}
$$

and $a_{n_{1}, n_{2}-2 n_{1}}$ are the Fourier coefficients of $f(q+2 p, p)$. This proves the claim.

It will prove convenient in our later discussion of quantum symmetries (section 3 ) to establish the following proposition.

Proposition 2.2. For $N \equiv 0 \bmod 4$ the matrix $\hat{B}$ has the chessboard form

$$
\hat{B}=\left(\begin{array}{cccccc}
* & 0 & * & 0 & \cdots & 0 \\
0 & * & 0 & * & \cdots & * \\
* & 0 & * & 0 & \cdots & 0 \\
0 & * & 0 & * & \cdots & * \\
\vdots & \vdots & \vdots & \vdots & \ddots & \vdots \\
0 & * & 0 & * & \cdots & *
\end{array}\right) \text {, }
$$

where * denotes non-zero (but not necessarily equal) entries. If $N \equiv 2 \bmod 4$ then $\hat{B}$ has the complementary form to (2.23) (i.e. with the 0 's and $*$ 's exchanging positions). For other values of $N$ all entries of $\hat{B}$ are non-zero in general.

Proof. We can simplify the expression for $\hat{B}$ by multiplying out the matrices in (2.16),

$$
\begin{aligned}
\hat{B}_{j k} & =\frac{1}{N} \sum_{\ell=0}^{N-1} \sum_{m=0}^{N-1} e_{N}(j \ell) e_{N}\left(\ell^{2}\right) \delta_{\ell m} e_{N}(-m k) \\
& =\frac{1}{N} \sum_{m=0}^{N-1} e_{N}\left(m^{2}+(j-k) m\right) \\
& =\frac{1}{N} e_{N}\left(-\frac{(j-k)^{2}}{4}\right) \sum_{m=0}^{N-1} e_{N}\left(\left(m+\frac{j-k}{2}\right)^{2}\right) .
\end{aligned}
$$

We now consider the parity of $j-k$. If $j-k$ is even, then $(j-k) / 2 \in \mathbb{Z}$ so by re-indexing the summation, we arrive at

$$
\hat{B}_{j k}=\frac{1}{N} e_{N}\left(-\frac{(j-k)^{2}}{4}\right) \sum_{n=0}^{N-1} e_{N}\left(n^{2}\right) .
$$

The summation is now a classical Gauss sum, and may be evaluated explicitly (see for example [5]). The result is

$$
\hat{B}_{j k}=\frac{1}{\sqrt{N}} \varepsilon_{N} e_{N}\left(-\frac{(j-k)^{2}}{4}\right)
$$


where $\varepsilon_{N}$ is given by

$$
\varepsilon_{N}:=\left\{\begin{array}{ccc}
1, & \text { if } N \equiv 1 & \bmod 4 \\
0, & \text { if } N \equiv 2 \bmod 4 \\
\text { i, } & \text { if } N \equiv 3 \bmod 4 \\
1+\mathrm{i}, & \text { if } N \equiv 0 & \bmod 4
\end{array}\right.
$$

On the other hand, if $j-k$ is odd, then by re-indexing the best we can do is

$$
\hat{B}_{j k}=\frac{1}{N} e_{N}\left(-\frac{(j-k)^{2}}{4}\right) \sum_{n=0}^{N-1} e_{N}\left(\left(n+\frac{1}{2}\right)^{2}\right) .
$$

Nevertheless, this summation can also be evaluated (see [5], exercise 1.23), giving

$$
\hat{B}_{j k}=\frac{1}{\sqrt{N}} \varepsilon_{N}^{\prime} e_{N}\left(-\frac{(j-k)^{2}}{4}\right)
$$

where $\varepsilon_{N}^{\prime}$ is given by

$$
\varepsilon_{N}^{\prime}:=\left\{\begin{array}{ccc}
\text { i, } & \text { if } N \equiv 1 & \bmod 4 \\
1+\mathrm{i}, & \text { if } N \equiv 2 & \bmod 4 \\
1, & \text { if } N \equiv 3 & \bmod 4 \\
0, & \text { if } N \equiv 0 & \bmod 4
\end{array}\right.
$$

So from (2.27) and (2.30) we see that for $N$ odd there are no zero entries, and if $N$ is even there are zero entries along the odds diagonal if $N \equiv 0 \bmod 4$ and along the even diagonals if $N \equiv 2 \bmod 4$.

\subsection{Quantising $R_{\beta}$ and $G_{\alpha}$}

The map $R_{\beta}$ is a translation on $\mathbb{T}^{2}$. Nevertheless simply setting $\ddagger\left\langle j\left|\hat{R}_{\beta}\right| k\right\rangle=e(\beta k) \delta_{j k}$ does not give a quantisation satisfying an Egorov estimate for arbitrary $f$. This is due to a "quantum discontinuity" at the line $q=0$ [24]. One possible resolution proposed in [40] was a quantisation in which $\beta$ is replaced by a rational approximation of the form $\beta_{N}=b_{N} / N$ such that $\beta_{N} \rightarrow \beta$ as $N \rightarrow \infty$. Making this choice, gives another exact Egorov estimate.

In fact for many applications, such as proving equidistribution of almost all eigenvectors (see corollary 2.5) it is only necessary to prove an Egorov estimate for a restricted class of observables. Two different approaches to this can be found in the literature; in [24] applied in the case of the quantum baker and sawtooth maps, and [39] in a more general setting. The method we outline below follows the spirit of [39]. To be precise, we can take observables that are supported away from the set $\{0\} \times \mathbb{T}$. In fact since the set $\{0,1 / 2\} \times \mathbb{T}$ is the set of discontinuity of the map $G_{\alpha}$ so it is convenient to quantise $R_{\beta}$ and $G_{\alpha}$ together, and take observables supported away from this set of discontinuity.

Let $H_{\alpha, \beta}:=R_{\beta} \circ G_{\alpha}$. We define the quantisation of $H_{\alpha, \beta}$ by $\hat{H}_{\alpha, \beta}$ which is a matrix with entries

$$
\left\langle j\left|\hat{H}_{\alpha, \beta}\right| k\right\rangle=e\left(-N\left(\beta\left\{\frac{k}{N}\right\}+\alpha\left\{\frac{k}{N}\right\} \theta\left(\frac{k}{N}\right)\right)\right) \delta_{j k}
$$

where $\{\cdot\}$ is the fractional part function. This quantisation respects the periodicity (2.9), since

$$
\hat{H}_{\alpha, \beta}|m N+\ell\rangle=\hat{H}_{\alpha, \beta}|\ell\rangle .
$$

$\ddagger$ We shall occasionally use the Dirac notation $\langle j|A| k\rangle$ to denote the $j k^{\text {th }}$ entry of a matrix $A$, for notational simplicity. 
In order to prove a semi-classical version of lemma 2.1 for $\hat{H}_{\alpha, \beta}$ we make use of the observation in [39] that the quantisation recipe (2.8) can be equivalently expressed as

$$
\hat{f}|\ell\rangle=\sum_{m \in \mathbb{Z}} b\left(\frac{\ell}{N}+\frac{m}{2 N}, m\right)|\ell+m\rangle,
$$

where $b(q, m)$ are the Fourier coefficients of the function $f$ expanded only in the $p$-variable,

$$
b(q, m):=\int_{0}^{1} f(q, p) e(-m p) \mathrm{d} p .
$$

(For a proof of this fact see [41].)

Lemma 2.3. Let $f \in C^{\infty}\left(\mathbb{T}^{2}\right)$ be compactly supported away from the set $\{0,1 / 2\} \times \mathbb{T}$. Then for any $R>0$,

$$
\left\|\hat{H}_{\alpha, \beta}^{-1} \hat{f} \hat{H}_{\alpha, \beta}-f \widehat{\circ H_{\alpha, \beta}}\right\|=\mathrm{O}_{R, f}\left(N^{-R}\right),
$$

as $N \rightarrow \infty$.

Proof. Define $V(x):=\beta\{x\}+\alpha\{x\} \theta(x)$. Then using (2.33),

$\hat{H}_{\alpha, \beta}^{-1} \hat{f} \hat{H}_{\alpha, \beta}|\ell\rangle=\sum_{m \in \mathbb{Z}} b\left(\frac{\ell}{N}+\frac{m}{2 N}, m\right) e\left(-N V\left(\frac{l}{N}\right)+N V\left(\frac{\ell+m}{N}\right)\right)|\ell+m\rangle$.

On the other hand, expanding the composed map in a Fourier series

$$
f \circ H_{\alpha, \beta}(q, p)=\sum_{m \in \mathbb{Z}} \tilde{b}(q, m) e(m p)
$$

the coefficients are

$$
\begin{aligned}
\tilde{b}(q, m) & =\int_{0}^{1} f(q, p+\beta+\alpha \theta(q)) e(-m p) \mathrm{d} p \\
& =e(m \beta+m \alpha \theta(q)) b(q, m) .
\end{aligned}
$$

Hence

$$
\begin{aligned}
\widehat{f \circ H_{\alpha, \beta}}|\ell\rangle & =\sum_{m \in \mathbb{Z}} \tilde{b}\left(\frac{\ell}{N}+\frac{m}{2 N}, m\right)|\ell+m\rangle \\
& =\sum_{m \in \mathbb{Z}} e\left(m \beta+m \alpha \theta\left(\frac{\ell}{N}+\frac{m}{2 N}\right)\right) b\left(\frac{\ell}{N}+\frac{m}{2 N}, m\right)|\ell+m\rangle .
\end{aligned}
$$

So from (2.35) and (2.37) it follows that

$$
\begin{gathered}
\left(\hat{H}_{\alpha, \beta}^{-1} \hat{f} \hat{H}_{\alpha, \beta}-\widehat{f \circ H_{\alpha, \beta}}\right)|\ell\rangle=\sum_{m \in \mathbb{Z}} b\left(\frac{\ell}{N}+\frac{m}{2 N}, m\right)\left(e\left(-N V\left(\frac{\ell}{N}\right)+N V\left(\frac{\ell+m}{N}\right)\right)\right. \\
\left.-e\left(m \beta+m \alpha \theta\left(\frac{\ell}{N}+\frac{m}{2 N}\right)\right)\right)|\ell+m\rangle,
\end{gathered}
$$

and by splitting the range of summation and applying the triangle inequality

$$
\begin{aligned}
\left.\| \hat{H}_{\alpha, \beta}^{-1} \hat{f} \hat{H}_{\alpha, \beta}-f \widehat{\circ H_{\alpha, \beta}}\right)|\ell\rangle \| \leqslant 2 \sum_{|m| \geqslant N^{1 / 2}}\left|b\left(\frac{\ell}{N}+\frac{m}{2 N}, m\right)\right| \\
\quad+\sum_{|m|<N^{1 / 2}} \mid e\left(-N V\left(\frac{\ell}{N}\right)+N V\left(\frac{\ell+m}{N}\right)\right) \\
-e\left(m \beta+m \alpha \theta\left(\frac{\ell}{N}+\frac{m}{2 N}\right)\right)|| b\left(\frac{\ell}{N}+\frac{m}{2 N}, m\right) \mid .
\end{aligned}
$$


To handle the first term in (2.39) we note that since $f \in C^{\infty}\left(\mathbb{T}^{2}\right)$ for any $R>0$ there exists a constant $C_{R}$ which does not depend on $q$ such that

$$
|b(q, m)| \leqslant \frac{C_{R}}{(1+|m|)^{2 R+2}} .
$$

Then

and

$$
\begin{aligned}
\left|b\left(\frac{\ell}{N}+\frac{m}{2 N}, m\right)\right| & \leqslant \frac{C_{R}}{(1+|m|)^{2 R+2}} \\
& \leqslant \frac{1}{(1+\sqrt{N})^{2 R}} \frac{C_{R}}{(1+|m|)^{2}} \\
& \leqslant \frac{1}{N^{R}} \frac{C_{R}}{(1+|m|)^{2}}
\end{aligned}
$$

$$
\sum_{|m| \geqslant N^{1 / 2}} \frac{1}{(1+|m|)^{2}}<\infty .
$$

For $m<N^{1 / 2}$ we note that for $N$ sufficiently large either $b\left(\frac{\ell}{N}+\frac{m}{2 N}, m\right)=0$ if $\ell / N$ is close to 0 or $1 / 2$ because of the restriction on the support of $f$, or otherwise

$$
\theta\left(\frac{\ell}{N}\right)=\theta\left(\frac{\ell}{N}+\frac{m}{2 N}\right)=\theta\left(\frac{\ell}{N}+\frac{m}{N}\right)
$$

and

$$
\left\{\frac{\ell}{N}\right\}=\frac{\ell}{N} \quad \text { and } \quad\left\{\frac{\ell+m}{N}\right\}=\frac{\ell+m}{N}
$$

In this case

$$
\begin{aligned}
e\left(-N V\left(\frac{\ell}{N}\right)\right. & \left.+N V\left(\frac{\ell+m}{N}\right)\right)=e\left(-\beta \ell-\alpha \ell \theta\left(\frac{\ell}{N}\right)+\beta(\ell+m)+\alpha(\ell+m) \theta\left(\frac{\ell+m}{N}\right)\right) \\
= & e\left(m \beta+m \alpha \theta\left(\frac{\ell}{N}+\frac{m}{2 N}\right)\right),
\end{aligned}
$$

so that for sufficiently large $N$ the second sum in (2.39) vanishes identically. The required estimate now follows easily.

We remark that the matrix $\hat{H}_{\alpha, \beta}$ can be written itself as a product $\hat{H}_{\alpha, \beta}=\hat{R}_{\beta} \hat{G}_{\alpha}$, where $\hat{R}_{\beta}$ and $\hat{G}_{\alpha}$ are the diagonal matrices

$$
\left\langle j\left|\hat{R}_{\beta}\right| k\right\rangle:=e\left(-N \beta\left\{\frac{k}{N}\right\}\right) \delta_{j k}
$$

and

$$
\left\langle j\left|\hat{G}_{\alpha}\right| k\right\rangle:=e\left(-N \alpha\left\{\frac{k}{N}\right\} \theta\left(\frac{k}{N}\right)\right) \delta_{j k},
$$

respectively defined to be the quantisations of the individual maps $R_{\beta}$ and $G_{\alpha}$.

It is now a simple matter to prove the Egorov estimate for the Casati-Prosen map.

Theorem 2.4. Let $f \in C^{\infty}\left(\mathbb{T}^{2}\right)$ be compactly supported away from the set $B^{-1}(\{0,1 / 2\} \times \mathbb{T})$. Then for any $R>0$,

$$
\left\|U_{\alpha, \beta}^{-1} \hat{f} U_{\alpha, \beta}-\widehat{f \circ F_{\alpha, \beta}}\right\|=\mathrm{O}_{R, f}\left(N^{-R}\right)
$$

as $N \rightarrow \infty$, where

$$
U_{\alpha, \beta}:=\hat{B} \hat{H}_{\alpha, \beta} .
$$


Proof. Let $h=f \circ B$, so that $f \circ F_{\alpha, \beta}=h \circ H_{\alpha, \beta}$. Then $h \in C^{\infty}\left(\mathbb{T}^{2}\right)$ since $f, B \in C^{\infty}\left(\mathbb{T}^{2}\right)$ and moreover $h$ is supported away from the set $\{0,1 / 2\} \times \mathbb{T}$. So by lemma 2.3 , for any $R>0$

$$
\left\|\hat{H}_{\alpha, \beta}^{-1} \hat{h} \hat{H}_{\alpha, \beta}-\widehat{h \circ H_{\alpha, \beta}}\right\|=\mathrm{O}\left(N^{-R}\right)
$$

as $N \rightarrow \infty$ where the implied constant can depend on $R$ and the choice of $f$. Now,

$$
\begin{aligned}
\left\|U_{\alpha, \beta}^{-1} \hat{f} U_{\alpha, \beta}-\widehat{f \circ F_{\alpha, \beta}}\right\|=\left\|\hat{H}_{\alpha, \beta}^{-1} \hat{B}^{-1} \hat{f} \hat{B} \hat{H}_{\alpha, \beta}-\widehat{f \circ F_{\alpha, \beta}}\right\| & \\
& \leqslant\left\|\hat{H}_{\alpha, \beta}^{-1} \hat{B}^{-1} \hat{f} \hat{B} \hat{H}_{\alpha, \beta}-\hat{H}_{\alpha, \beta}^{-1} \widehat{f \circ B} \hat{H}_{\alpha, \beta}\right\|+\left\|\hat{H}_{\alpha, \beta}^{-1} \hat{h} \hat{H}_{\alpha, \beta}-\widehat{h \circ H_{\alpha, \beta}}\right\| \\
& =\left\|\hat{B}^{-1} \hat{f} \hat{B}-\widehat{f \circ B}\right\|+\mathrm{O}\left(N^{-R}\right) \quad \text { from }(2.50) \\
& =\mathrm{O}\left(N^{-R}\right),
\end{aligned}
$$

by lemma 2.1 .

The proof of an Egorov estimate and good control over the singularities are all we need to prove equidistribution of almost all eigenvectors, assuming ergodicity of the classical map. Methods for handling singularities are based on ideas from [48] and were adapted to maps in [24]. An equivalent approach was presented in [39]. Thus we have the following Schnirelman theorem, stated as a corollary.

Corollary 2.5. Let $\alpha$ and $\beta$ be such that the map $F_{\alpha, \beta}$ is ergodic with respect to Lebesgue measure $\mu$. Then for each $N$ there exists a subset $J_{N} \subseteq\{0, \ldots, N-1\}$ of density one (i.e. satisfying $\left.\lim _{N \rightarrow \infty} \# J_{N} / N=1\right)$, such that for any observable $f \in C^{\infty}\left(\mathbb{T}^{2}\right)$,

$$
\lim _{N \rightarrow \infty}\left\langle\psi_{j_{N}}|\hat{f}| \psi_{j_{N}}\right\rangle=\int_{\mathbb{T}^{2}} f(q, p) \mathrm{d} \mu
$$

where $j_{N} \in J_{N}$, and $\left|\psi_{j}\right\rangle$ is a normalised basis of eigenvectors of $U_{\alpha, \beta}$.

Proof. We refer the reader to the proof mutatis mutandis of corollary 8.2 in [39] and references therein.

We note that the existence of such $\alpha$ and $\beta$ in corollary 2.5 is, to the best of our knowledge, still a conjecture.

\section{Symmetries}

Before studying the spectral statistics of the Casati-Prosen map, it is necessary to consider the symmetries of the map. An important question is whether or not the quantised map admits an anti-unitary symmetry, such as quantum time-reversibility. But also other symmetries can lead to non-generic spectral statistics, and we demonstrate an example of such a symmetry in subsection 3.2 .

\subsection{Time reversal symmetry}

A map $M$ has an anti-canonical symmetry under the map $\tilde{\tau}$ if the following relation holds

$$
\tilde{\tau} \circ M \circ \tilde{\tau}=M^{-1}, \quad \text { with } \tilde{\tau}^{2}=\mathrm{id} .
$$

The most well known example of such a symmetry is time reversal $\tau$ which is the map defined by

$$
\tau:\left(\begin{array}{c}
q \\
p
\end{array}\right) \mapsto\left(\begin{array}{c}
q \\
-p
\end{array}\right) .
$$


In fact the individual maps $B, R_{\beta}$ and $G_{\alpha}$ are time reversal symmetric;

$$
\begin{aligned}
& \tau \circ B \circ \tau=B^{-1}, \\
& \tau \circ R_{\beta} \circ \tau=R_{-\beta}=R_{\beta}^{-1}, \\
& \tau \circ G_{\alpha} \circ \tau=G_{-\alpha}=G_{\alpha}^{-1},
\end{aligned}
$$

as may be easily checked.

The Casati-Prosen map is not time reversal symmetric, but does admit an anti-canonical symmetry given by

$$
\tilde{\tau}:=R_{-\beta / 2} \circ G_{-\alpha / 2} \circ \tau \circ G_{\alpha / 2} \circ R_{\beta / 2} .
$$

This follows from the fact that the symmetrised map

$$
F_{\alpha, \beta}^{\mathrm{sym}}:=R_{\beta / 2} \circ G_{\alpha / 2} \circ B \circ G_{\alpha / 2} \circ R_{\beta / 2}
$$

is time reversal symmetric - an easy consequence of (3.52)-(3.54).

The quantum analogue of $\tau$ is $T$ the complex conjugation operator,

$$
T \psi=\bar{\psi},
$$

so we define the quantum version of $\tilde{\tau}$ to be

$$
\tilde{T}:=\hat{R}_{-\beta / 2} \hat{G}_{-\alpha / 2} T \hat{G}_{\alpha / 2} \hat{R}_{\beta / 2} .
$$

Then in an analogous way it follows that $\tilde{T}$ is an anti-unitary symmetry of $U_{\alpha, \beta}$, i.e.

$$
\tilde{T} U_{\alpha, \beta} \tilde{T}=U_{\alpha, \beta}^{-1} .
$$

The relevance of anti-unitary symmetry for quantum maps is that their presence, or absence, determines the universality class for the Bohigas-Gianonni-Schmit conjecture. In generic quantum maps with an anti-unitary symmetry, the semi-classical spectral statistics are conjectured to be those of the circular orthogonal ensemble of random matrix theory [38], while in systems where anti-unitary symmetry is absent, the statistics should be the same as those of the circular unitary ensemble [42].

\subsection{Other symmetry}

The rotation $R_{1 / 2}$ commutes with $F_{\alpha, \beta}$. We investigate the implications of this for the quantised map. By (2.46) we see that $\hat{R}_{1 / 2}$ has a particularly simple matrix representation; in the canonical basis the elements are

$$
\left\langle j\left|\hat{R}_{1 / 2}\right| k\right\rangle=(-1)^{k} \delta_{j k} .
$$

Also $\hat{R}_{1 / 2}$ is self-inverse; $\hat{R}_{1 / 2}^{2}=I_{N}$ where $I_{N}$ is the $N \times N$ identity matrix. Thus, by (3.59), conjugating any matrix $A$ with $\hat{R}_{1 / 2}$ gives a result with entries

$$
\left\langle j\left|\hat{R}_{1 / 2}^{-1} A \hat{R}_{1 / 2}\right| k\right\rangle=\left\langle j\left|\hat{R}_{1 / 2} A \hat{R}_{1 / 2}\right| k\right\rangle=(-1)^{j+k} A_{j k} .
$$

Since $\hat{H}_{\alpha, \beta}$ is diagonal, for $N$ even $U_{\alpha, \beta}$ inherits the chessboard structure of $\hat{B}(2.23)$ in proposition 2.2. This means that if $N$ is even with $N \equiv 0 \bmod 4$ then $\hat{R}_{1 / 2}$ commutes with $U_{\alpha, \beta}$ and if $N \equiv 2 \bmod 4$ then $\hat{R}_{1 / 2}$ and $U_{\alpha, \beta}$ anticommute. If $N$ is odd then $\hat{R}_{1 / 2}$ and $U_{\alpha, \beta}$ do not commute.

We consider first the case that $N \equiv 0 \bmod 4$. Then $\hat{R}_{1 / 2}$ and $U_{\alpha, \beta}$ have a joint basis of eigenvectors. Since $\hat{R}_{1 / 2}$ is self-inverse, the only eigenvalues are \pm 1 . So the eigenvectors (and 
correspondingly eigenvalues) $\psi_{j}$ of $U_{\alpha, \beta}$ can be divided into two parity classes according to whether $\hat{R}_{1 / 2} \psi_{j}=\psi_{j}$ or $\hat{R}_{1 / 2} \psi_{j}=-\psi_{j}$. Eigenvectors in the first class will have the form

$$
\psi_{j}=(*, 0, *, 0, \ldots, 0)
$$

and eigenvectors of the second class have the form

$$
\psi_{j}=(0, *, 0, *, \ldots, *) .
$$

In the case that $N \equiv 2 \bmod 4$, we have

$$
\hat{R}_{1 / 2}^{-1} U_{\alpha, \beta} \hat{R}_{1 / 2}=-U_{\alpha, \beta},
$$

so that $\hat{R}_{1 / 2}$ commutes with $U_{\alpha, \beta}^{2}$. If $\mathrm{e}^{\mathrm{i} \phi_{j}}$ is an eigenvalue of $U_{\alpha, \beta}$ with eigenvector $\psi_{j}$ then,

$$
U_{\alpha, \beta} \hat{R}_{1 / 2} \psi_{j}=-\hat{R}_{1 / 2} U_{\alpha, \beta} \psi_{j}=-\mathrm{e}^{\mathrm{i} \phi_{j}} \hat{R}_{1 / 2} \psi_{j} .
$$

So $\hat{R}_{1 / 2} \psi_{j}$ is an eigenvector of $U_{\alpha, \beta}$ with eigenvalue $-\mathrm{e}^{\mathrm{i} \phi_{j}}$. This means that eigenvalues of $U_{\alpha, \beta}$ again come in pairs separated by an angle $\pi$. Another consequence is that the eigenspace of the eigenvalue $\mathrm{e}^{2 \mathrm{i} \phi_{j}}$ of $U_{\alpha, \beta}^{2}$ is spanned by the vectors $\psi_{j}$ and $\hat{R}_{1 / 2} \psi_{j}$. So if $\chi$ is in this eigenspace then

$$
\chi=c_{1} \psi_{j}+c_{1} \hat{R}_{1 / 2} \psi_{j}
$$

for some constants $c_{1}, c_{2}$. Since $\hat{R}_{1 / 2}$ commutes with $U_{\alpha, \beta}^{2}$ then $\hat{R}_{1 / 2} \chi= \pm \chi$, and this fixes the ratio $c_{1} / c_{2}= \pm 1$. Then $\chi$ is necessarily of the form (3.61) or (3.62) described above.

\section{Eigenvalues}

Since $U_{\alpha, \beta}$ has an anti-unitary symmetry, according to the Bohigas-Giannoni-Schmit conjecture [12], if the Casati-Prosen map behaves generically we would expect that the statistics of eigenvalues of $U_{\alpha, \beta}$ converge to the statistics of the circular orthogonal ensemble of random matrix theory (COE), as $N \rightarrow \infty$. On the other hand, recent studies [30] of similar maps have revealed spectral statistics which are of intermediate type or deviate in some manner from random matrix theory.

The eigenvalue statistics that we consider are the nearest-neighbour spacing distribution and the pair-correlation form factor. If the matrix $U_{\alpha, \beta}$ has the set of eigenvalues $\left\{\mathrm{e}^{\mathrm{i} \phi_{j}}: j=0, \ldots, N-1\right\}$ (each eigenvalue counted according to its multiplicity) then we consider the re-scaled angles

$$
\lambda_{j}:=\frac{N}{2 \pi} \phi_{j}
$$

where we first sort the angles so that $0 \leqslant \phi_{0} \leqslant \cdots \leqslant \phi_{N-1}<2 \pi$. The average spacing of the $\lambda_{j}$ is then 1 . The nearest neighbour density is defined as the probability density $P(s)$ (if it exists) such that

$$
\lim _{N \rightarrow \infty} \frac{1}{N} \sum_{j=1}^{N-1} h\left(\lambda_{j}-\lambda_{j-1}\right)=\int_{0}^{\infty} h(s) P(s) \mathrm{d} s
$$

for some class of test functions $h$. For random matrices chosen from the COE, the corresponding density $P^{\mathrm{COE}}(s)$ is well-known [38]. The closed form is complicated, given in terms of integrals of Painlevé functions [27], but is known to be numerically well-approximated by the Wigner surmise given by

$$
P^{\mathrm{COE}}(s) \approx \frac{\pi s}{2} e^{-\pi s^{2} / 4} .
$$

The pair correlation form factor is defined to be the density $K_{2}(\tau)$ such that

$$
\lim _{N \rightarrow \infty} \int_{-\infty}^{\infty} K_{2}(\tau, N) h(\tau) \mathrm{d} \tau=\int_{-\infty}^{\infty} K_{2}(\tau) h(\tau) \mathrm{d} \tau,
$$


if it exists for some class of test functions $h$, where $K_{2}(\tau, N)$ is defined by

$$
K_{2}(\tau, N):=\frac{1}{N}\left|\sum_{n=0}^{N-1} e\left(\lambda_{n} \tau\right)\right|^{2}
$$

At rational times $\tau=n / N$ it follows that $K_{2}(\tau, N)=\frac{1}{N}\left|\operatorname{tr}\left(U_{\alpha, \beta}^{n}\right)\right|^{2}$. We note that $K_{2}(\tau)$ is not a probability density. For the COE, this quantity can be calculated exactly [38],

$$
K_{2}^{\mathrm{COE}}(\tau)= \begin{cases}2|\tau|-|\tau| \log (1+2|\tau|), & \text { for }|\tau| \leqslant 1 \\ 2-|\tau| \log \left(\frac{2|\tau|+1}{2|\tau|-1}\right), & \text { for }|\tau|>1\end{cases}
$$

The nearest-neighbour spacing density is a local statistic, in the sense that it gives a measure of correlations only on the scale of the mean separation. The pair correlation form factor, on the other hand, measures correlations on all scales.

In figure 2 we show these statistics calculated numerically for a realisation of the quantum Casati-Prosen map with $\alpha$ and $\beta$ irrational, together with the exact curves calculated for the COE. The size of the matrix in the numerical study is $N=7001$. For the form factor, the data has been averaged, so that each data point in the plot is the average of 10 computed values. For both statistics good agreement is seen, suggesting that for these choices, the Casati-Prosen map behaves generically with respect to the Bohigas-Gianonni-Schmit conjecture. For other choices of irrational and linearly independent $\alpha$ and $\beta$ similar agreement with COE was observed. The experiment was also repeated with even $N$, in which case the symmetry discussed in section 3.2 means that it is correct to consider the statistics of the subspectra of eigenvalues seperately. Within these subspectra, the statistics also conformed well to COE predictions, although the data is less "clean" because the effective value of $N$ is reduced by half.
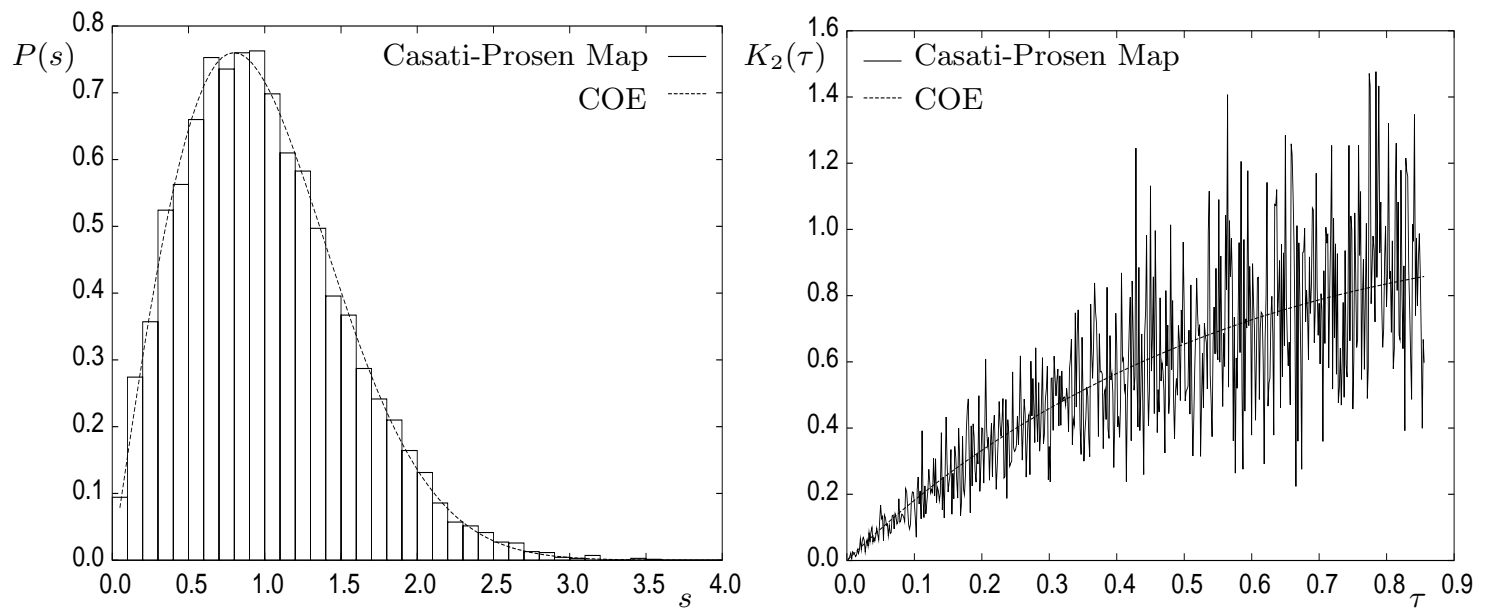

Figure 2. Spectral statistics for $\alpha=(\sqrt{5}-1) / 2$ and $\beta=\sqrt{2}, N=7001$. On the left is the nearest neighbour density, and on the right the form factor.

In figure 3 we show an example of a realisation with $\beta=0$ and $\alpha$ irrational, also for $N=7001$. This numerical data does not follow the COE curves, neither for the nearest-neighbour spacing, nor for the form factor. The data appears to give spectral statistics that are "between" those of the COE and those for independent random events of a Poisson process. Such statistics are called intermediate statistics [9].

At this point, it would be interesting to see how these statistics behave as $N$ is increased further§. On decreasing $N$ we notice that the intermediate statistics become "further" from COE.

$\S$ We thank an anonymous referee for suggesting this. 
In particular the intercept $K_{2}(0)$ moves further from the COE point $K_{2}^{\mathrm{COE}}(0)=0$. This at least suggests the possibility that for much higher values of $N$, these statistics approach those of the COE, but at $N=7001$ we have reached the limits of computation with technology available to us, and have no way of checking further.

We have fitted the statistics in figure 3 to the ansatz [10] for the nearest-neighbour density,

$$
P^{\text {int }}(s)=\frac{b^{b}}{\Gamma(b)} s^{b-1} \mathrm{e}^{-b s},
$$

with parameter $b$. For example, $b=2$ gives the semi-Poisson distribution, and $b=1$ gives the exponential density of the Poisson process. We have found that the fitted parameter $b$ is different for different irrational $\alpha$, and for fixed $\alpha$, different values of $N$ give different values of $b$.
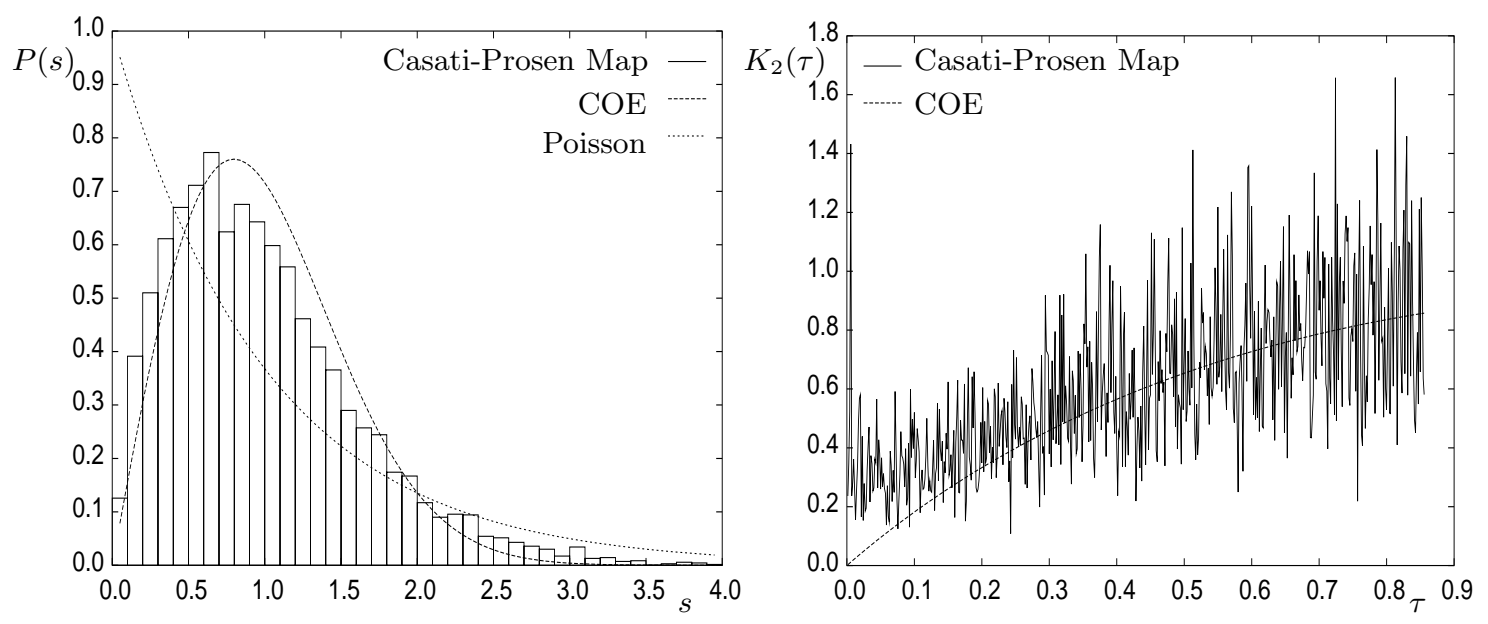

Figure 3. Spectral statistics for $\alpha=(\sqrt{5}-1) / 2$ and $\beta=0, N=7001$. On the left is the nearest neighbour density, and on the right the form factor.

Intermediate statistics are not new, and have been much studied in recent years, particularly when arising out of certain billiards in rational polygons $[9,18,10,11,31]$. Intermediate statistics conforming to the distribution (4.66) were found in [30], for a map equivalent to the triangle map with $\alpha=0$ and $\beta$ rational.

Also in [30] for $\beta$ irrational, deviations from random matrix theory were found for certain values of $N$. To be specific we define the parameter $\varepsilon(\beta)$ by

$$
\varepsilon(\beta):=\inf _{m \in \mathbb{Z}}\{|N \beta-m|\} .
$$

For choices of $N$ such that $\varepsilon(\beta)=\mathrm{O}\left(N^{-1 / 2}\right)$ as $N \rightarrow \infty$ highly non-random matrix spectral statistics were observed and explained using perturbation theory. The deviations in this case are not of intermediate type but something much more singular. Since such bad $N$ can occur infinitely often (see below) there is not a limiting density for the nearest neighbour spacing distribution of random matrix type in this case.

We recall that a vector $\boldsymbol{\alpha}=\left(\alpha_{1}, \ldots, \alpha_{k}\right) \in \mathbb{R}^{k}, k \geqslant 1$, is said to be badly approximable if there exists a constant $C>0$ such that

$$
\max _{1 \leqslant i \leqslant k}\left\{\inf _{m \in \mathbb{Z}}\left\{\left|n \alpha_{i}-m\right|\right\}\right\}>\frac{C}{n^{1 / k}} .
$$

The exponent $1 / k$ in $(4.68)$ is the best possible. Badly approximable vectors exist: see [25] for a proof. 
In the case $k=1$ no matter what choice of $\beta$ is made, there are always infinitely many choices of $N$ such that $\varepsilon(\beta)$ is arbitrarily much smaller than $1 / \sqrt{N}$. However the situation is different if we have a pair of parameters to simultaneously control.

Consider $\alpha, \beta \neq 0$ and independently irrational. If we chose $(\alpha, \beta)$ to be a badly approximable pair in the sense of (4.68), the quantities $\varepsilon(\alpha) \sqrt{N}$ and $\varepsilon(\beta) \sqrt{N}$ are both bounded below by a non-zero constant, and cannot become arbitrarily small. This could account for the robust results found for various values of $N$, taking into account symmetries. It appears as though the weakly chaotic action of the cutting, and shearing is sufficient to "break" the number theoretical properties of the skew-translations leading to non random-matrix spectral statistics. In the same way as a non-linear perturbation of the cat maps [4] breaks the number theoretical symmetries leading to non-generic spectral statistics in the unperturbed maps [33, 36]. However we emphasise that in the case of cat maps, both the perturbed and unperturbed maps are strongly chaotic. The triangle map does not possess such strong chaotic properties.

For $\beta=0, \alpha$ irrational, there are infinitely many choices of $N$ with small $\varepsilon(\alpha) \sqrt{N}$. If we group together subsequences of $N$ with $\varepsilon(\alpha) \sqrt{N}$ approximately constant, then the value of the parameter $b$ giving the best fit is approximately the same for most of the elements within each grouping. However there appears to be a small number of values of $N$ in each group, for which the fitting parameter is significantly different to the others. This points to some other number theoretical effect for which we do not currently have an explanation.

We note that in the case $k=1$ the most badly approximable numbers are the quadratic integers, which have periodic (and hence bounded) partial fraction coefficients. For the case $k=2$ less is understood though in [35] it is conjectured that the "most" badly approximable pair of irrationals $\|$ is $\left(x_{0}^{-1}, x_{0}^{-2}\right)$ where $x_{0}$ is the unique real root of the polynomial

$$
x^{3}-x-1 .
$$

We make use of this observation by picking $\left(x_{0}^{-1}, x_{0}^{-2}\right)$ as the parameter pair $(\alpha, \beta)$ for some numerical simulations in the next section. As we will see, it turns out that the eigenvector statistics appear to be much more sensitive to the approximability of parameters than the eigenvalues.

\section{Eigenvectors}

We also investigate the eigenvector statistics of the Casati Prosen map. Although the BohigasGiannoni-Schmit conjecture refers only to eigenvalues, there is some evidence to suggest that generic quantum maps also have eigenvector statistics which agree with those of the appropriate ensemble of random matrices. For example in [1] good agreement was seen between the value distribution of eigenvectors of perturbed cat maps, and random matrix theory. In [37] the nodal domain distribution, also of the eigenvectors of perturbed cat maps, was seen to agree well with the corresponding random matrix model.

Since, for example, the nodal domain distribution, is only defined for real eigenvectors, we considered for our numerical results the eigenvectors of the symmetrised map $U_{\alpha, \beta}^{\text {sym }}$. These eigenvectors are related to the eigenvectors of $U_{\alpha, \beta}$ by a linear transformation, but because the quantised operator $U_{\alpha, \beta}^{\mathrm{sym}}$ is unitary and symmetric, its eigenvectors have real components.

Given a (normalised) eigenvector $\mathbf{c}=\left(c_{0}, \ldots, c_{N-1}\right)$ of a matrix drawn at random from one of the circular ensembles, the value distribution is the probability distribution $p(\eta)$ (if it exists) such that

$$
\lim _{N \rightarrow \infty} \mathbb{E}\left(h\left(\left|c_{0}\right|^{2}\right)\right)=\int_{0}^{\infty} h(\eta) p(\eta) \mathrm{d} \eta .
$$

(Of course the index 0 in the left hand side of (5.70) could be replaced by any other index.) The expectation is with respect to the probability measure of the random matrix ensemble. For the 
$\mathrm{COE}$ it is the uniform measure supported on the surface of an $N$-dimensional unit sphere [14, 32]. This gives that the density for the value distribution of eigenvectors of the COE is

$$
p^{\mathrm{COE}}(\eta)=\frac{1}{\sqrt{2 \pi \eta}} \mathrm{e}^{-\eta / 2},
$$

known as the Porter-Thomas density. In figure 4 we present the numerical calculations of the value distribution for the components of one of the eigenvectors of the quantum Casati-Prosen map for matrices of size $N=4001$. In fact, in both regimes $\beta \neq 0$ and $\beta=0$, we get good agreement with the COE curves. Because of the simplicity of the assumptions that lead to the derivation of (5.71) it appears that this statistic is too crude to differentiate between random matrix behaviour, and the eigenvectors of non-generic unitary matrices. We also numerically measured the rate of convergence to the limiting density as $N$ increases and found that this also does not indicate any clear difference in the two regimes.
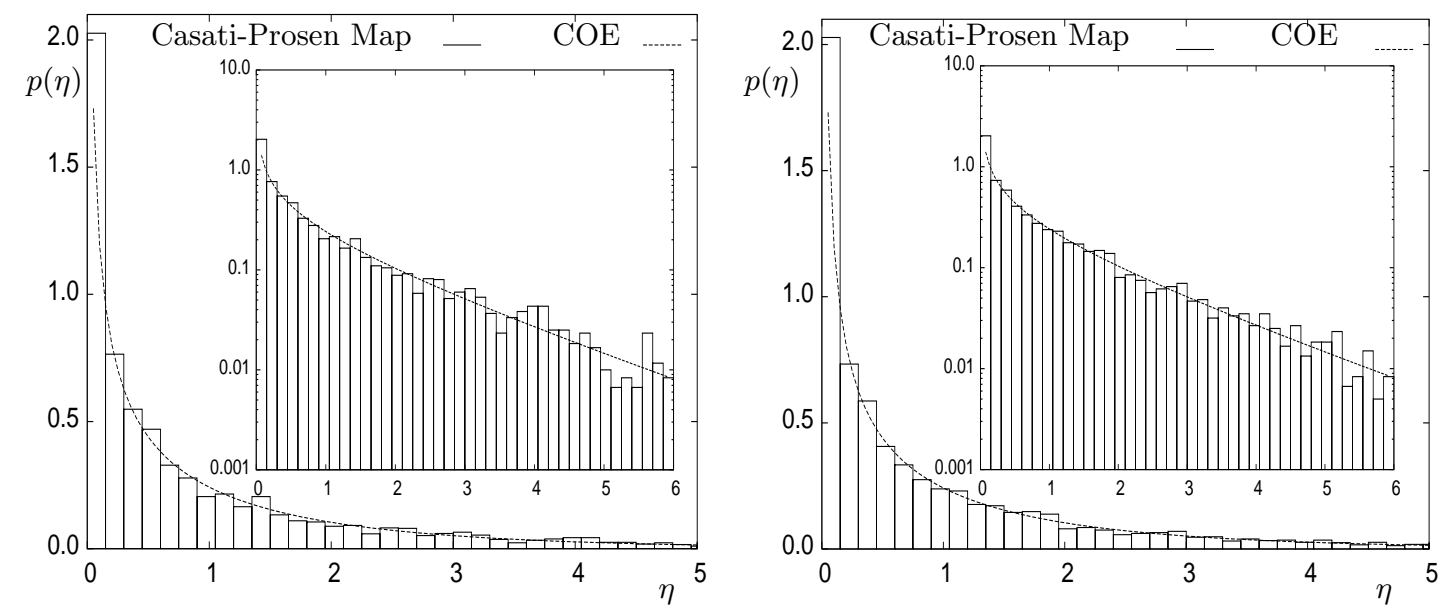

Figure 4. Value distribution of eigenvectors for $\alpha=(\sqrt{5}-1) / 2$ and $\beta=\sqrt{2}$ (left) and $\alpha=(\sqrt{5}-1) / 2$ and $\beta=0$ (right), at $N=4001$. The inset plots are of the same data on a log-normal scale.

The number of nodal domains has recently been proposed as an interesting eigenvalue statistic. Originally defined in the context of billiards [8], a nodal domain is a region where an eigenfunction has constant sign. The distribution of the normalised number of such domains over all eigenfunctions distinguishes between systems that are classically integrable, and those which are chaotic in the large wavenumber limit. This statistic was extended in a natural way to quantum maps [37] where the number of nodal domains is defined to be the number of sets of consecutive eigenvector components with the same sign. In fact this quantity is given by the formula

$$
\nu=\frac{1}{2} \sum_{j=0}^{N-1}\left(1-\operatorname{sign}\left(c_{j}\right) \operatorname{sign}\left(c_{j+1}\right)\right) .
$$

Following [37] we define the nodal domain density $V(N, x)$ by

$$
\frac{1}{N} \sum_{n=0}^{N-1} h\left(\frac{\nu_{n}}{N}\right)=\int_{-\infty}^{\infty} h(x) V(N, x) \mathrm{d} x,
$$

where $\nu_{n}$ is the number of nodal domains of the $n^{\text {th }}$ eigenvector. The expected value of the nodal domain distribution approaches $1 / 2$ so it is convenient to rescale, and look at the quantity

$$
\tilde{V}(N, h):=\frac{1}{N} \sum_{n=0}^{N-1} h\left(\sqrt{N}\left(\frac{\nu_{n}}{N}-\frac{1}{2}\right)\right) .
$$


In [37] it was proved that for random matrix eigenvalues from the COE, the limiting distribution of the rescaled nodal domains is that of a normal random variable with mean 0 and variance $1 / 4$, as a consequence of the central limit theorem of probability, i.e.

$$
\lim _{N \rightarrow \infty} \tilde{V}(N, h)=2 \int_{-\infty}^{\infty} h(x) \mathfrak{n}(2 x) \mathrm{d} x
$$

where $\mathfrak{n}(x):=\mathrm{e}^{-x^{2} / 2} / \sqrt{2 \pi}$ is the density of a standard normal random variable. For perturbed cat maps, the nodal domain distribution was seen to agree with (5.75). For unperturbed cat maps, the distribution also appeared to be Gaussian, but with a different variance to that in the random matrix case.

To investigate this statistic we calculated the distribution of number of nodal domain of eigenvectors of the Casati-Prosen map for several odd values of $N$ around $N=3760$ and plotted the average distribution. In figure 5 we show this data for the parameters $(\alpha, \beta)$ chosen to be the badly-approximable pair $\left(x_{0}^{-1}, x_{0}^{-2}\right)$. For single values of $N$ the data were not so smooth, necessitating the averaging. This particular choice of parameter pair gives the curve closest to the COE distribution, but even here there is some deviation. The data fit better to a Gaussian with variance $\approx 0.22$ rather than the $1 / 4$ predicted by random matrix theory. This is seen most clearly in the plot of the integrated density (figure 5:right). For the values of $N$ that we looked at, statistical indicators such as the sample variance still vary considerably, suggesting that we have still not yet reached the limiting regime. To check if one really does get convergence to COE for this statistic would therefore require tests at larger $N$. We note that if one really has convergence to the distribution for COE it must be slower than in the case of perturbed cat maps for which better agreement was noted for smaller values of $N$ [37].

For other (presumable less well-approximable) pairs of parameters $\alpha, \beta$ but still irrational and linearly independent the nodal domain density is better fitted by a Gaussian with variance different much further from $1 / 4$. For example with $\alpha=\frac{\sqrt{5}-1}{2}, \beta=\sqrt{2}$, the sample variance is $\approx 0.8$, at $N=4001$. If there is really convergence to random matrix theory, it must be even slower for these values of paramters. It is in this sense that we note that the eigenvector statistics are more sensitive to the approximability of the parameters $\alpha$ and $\beta$.
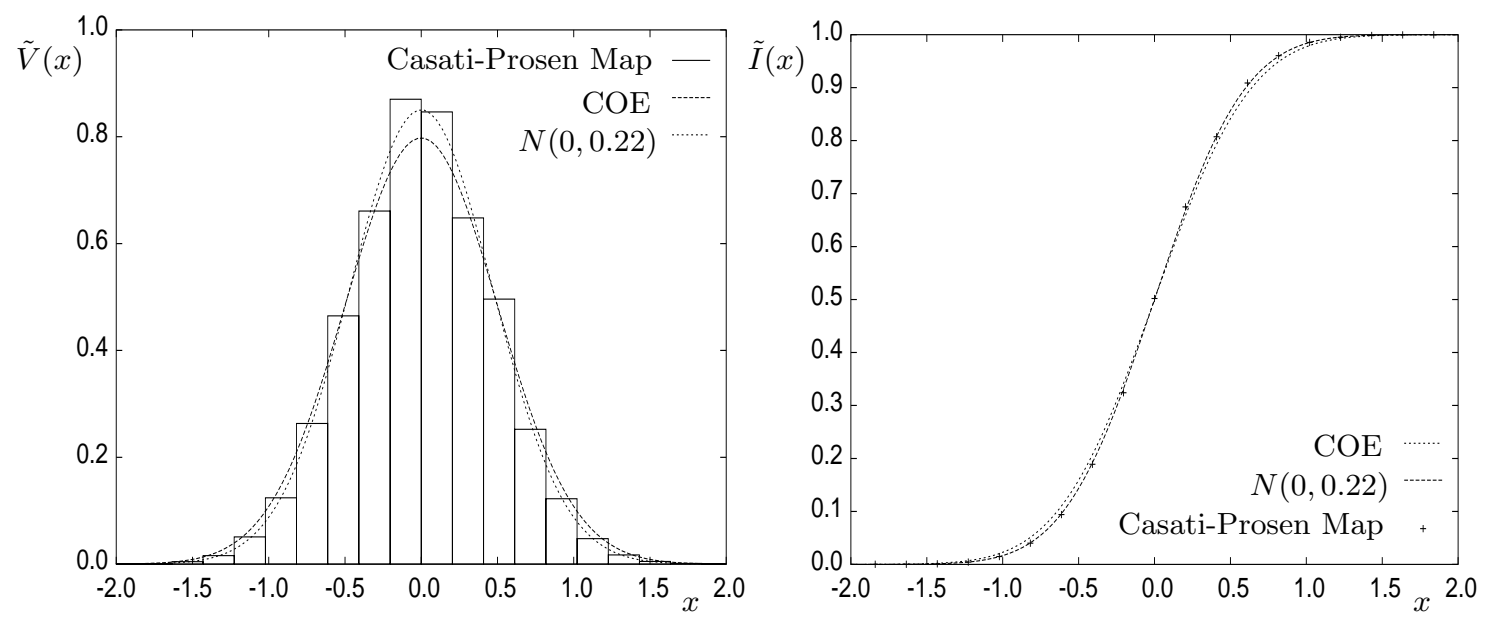

Figure 5. Averaged nodal domain density for $\alpha=x_{0}^{-1}$ and $\beta=x_{0}^{-2}$ (left) and the corresponding integrated density (right), $\tilde{I}(x):=\int_{-\infty}^{x} \tilde{V}(y) \mathrm{d} y$. We include the curves for COE, and a Gaussian distribution with variance 0.22 , denoted $N(0,0.22)$.

In the cases with $\beta=0$, the picture is somewhat different. The variance of the nodal domain distribution does not appear to be stablising as $N \rightarrow \infty$. In figure 6 we plot the integrated density for the Casati-Prosen map with $\alpha=x_{0}^{-1}$ and $\beta=0$, for different values of $N$. It is seen clearly 
that the density is spreading out as $N$ increases. We computed the sample variance of the density $V(N, x)$ for $\alpha=x_{0}^{-1}$ and $\alpha=\frac{\sqrt{5}-1}{2}$. In both cases we found the sample variance to vary like $N^{-\delta}$ with $\delta$ numerically calculated to be $\approx 0.7$. For a limiting density to exist we would have $\delta=1$.

We can speculate that this result is related to the classical decay of correlations for the underlying map. In [26] a study was made of the variance $\sigma^{2}$ of the distribution of fluctuations of diagonal matrix elements $\langle\psi|\hat{f}| \psi\rangle$, about the mean. In this work, the scaling of the variance was related to the classical decay of correlations. For non-hyperbolic systems the correct scaling was found to be $\sigma^{2} \propto \hbar^{\delta}$ for classical decay of correlations $\propto t^{-\gamma}, \gamma \neq 1$. In the case $\gamma>1$, or fasterthan-algebraic decay, we have $\delta=1$. For $\gamma<1$ we have $\delta<1$. Notice that since $\hbar=(2 \pi N)^{-1}$ for quantum maps this implies, in situations with fast decay of correlations such as perturbed cat maps and (conjecturally) the Casati-Prosen map with non-zero $\alpha$ and $\beta$, the quantity $\langle\psi|\hat{f}| \psi\rangle$ should be scaled by $\sqrt{N}$ to get a non-trivial limiting density. This is what one would naïvely expect since there are $N$ terms in the inner-product, each proportional to $N^{-1}$ (by normalisation). However, since for $\beta=0$ we expect that there is no decay of correlations, scaling by $\sqrt{N}$ should not lead to a non-trivial limiting density. Indeed we might expect that the density distribution becomes wider as the variance increases as $N \rightarrow \infty$.

To relate this to nodal domain distribution we can consider taking $f$ to be the observable $e(k p)$ for $k \in \mathbb{Z}$, in which case we get $\hat{f}=T(0, k)=t_{2}^{k}$. So for real eigenvectors,

$$
\langle\psi|\hat{f}| \psi\rangle=\sum_{j=0}^{N-1} \psi(j) \psi(j+k)
$$

which is the correlation between eigenvector components, and the component $k$ places away. For nodal domains, we are interested in a sum of the form, cf. (5.72),

$$
\sum_{j=0}^{N-1} \operatorname{sign}(\psi(j) \psi(j+1)) .
$$

Note the similarity to (5.76) with $k=1$. We might expect that correlations which cause the central limit theorem to fail in (5.77) are reflected by the correlations in (5.76). Thus we might expect some coincidence between the rate of growth of the variances in the sums (5.76) and (5.77).

As observed in [37] perturbed cat maps have nodal domain distribution which conforms well to random matrix theory, and by [26], the conjectural decay of correlations of $t^{-3 / 2}$ puts the CasatiProsen map with $\alpha$ and $\beta$ non-zero into the same scaling regime, so it would not be so surprising that the nodal domain distribution converges to the random matrix result. In the case $\beta=0$ we might expect that the nodal domain distribution becomes wider as $N$ increases, even after scaling, which agrees with our observations.

\section{Acknowledgments}

This work has been financially supported by the European Commission under the Research Training Network (Mathematical Aspects of Quantum Chaos) HPRN-CT-2000-00103 of the IHP Programme, and EPSRC research grant GR/R67279/01 (SO'K). The numerical computations were performed on an Alpha Workstation funded by a Royal Society research grant.

SO'K wishes to thank the University of Bologna for hospitality while the major part of this research was carried out.

We thank Arnd Bäcker for useful comments.

\section{References}

[1] Bäcker A 2003 Numerical aspects of eigenvalue and eigenfunction computations for chaotic quantum systems. In [22] pp. 91-144 


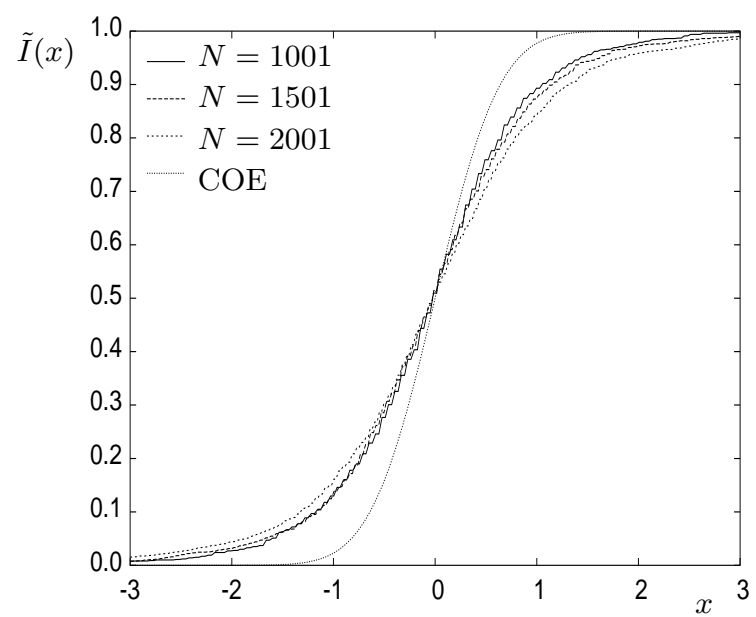

Figure 6. The scaled integrated density of nodal domains for $\alpha=x_{0}^{-1}$ and $\beta=0$ for varying $N$.

[2] Bäcker A and Haag G 1999 Spectral statistics for quantized skew translations on the torus J. Phys. A 32 L393-L398

[3] Bäcker A, Schubert R and Stifter P 1998 Rate of quantum ergodicity in Euclidean billiards Phys. Rev. E 57 5425-5447. Erratum 1998 Phys. Rev. E 585192.

[4] Basilio de Matos M and Ozorio de Almeida A M 1995 Quantization of Anosov maps Ann. Phys. 237 46-65

[5] Berndt B C, Evans R J and Williams K S 1998 Gauss and Jacobi sums (volume 21 of the Canadian Mathematical Society series of monographs and advanced texts, Wiley)

[6] Berry M V 1977 Regular and irregular semiclassical wavefunctions J. Phys. A 10 2083-2091

[7] Berry M V and Tabor M 1977 Level clustering in the regular spectrum Proc. Roy. Soc A 356 375-394

[8] Blum G, Gnutzmann S and Smilansky U 2002 Nodal domain statistics: a criterion for quantum chaos Phys. Rev. Lett. 88144101

[9] Bogomolny E, Gerland U and Schmit C 1999 Models of intermediate spectral statistics Phys. Rev. E 59 R1315-R1318

[10] Bogomolny E, Gerland U and Schmit C 2001 Short-range plasma model for intermediate spectral statistics Eur. Phys. J. B 19 121-132

[11] Bogomolny E, Giraud O and Schmit C 2001 Periodic orbits contribution to the two-point correlation form factor for pseudo-integrable systems Commun. Math. Phys. 222 327-369

[12] Bohigas O, Giannoni M-J and Schmit C 1984 Characterization of chaotic quantum spectra and universality of level fluctuation laws Phys. Rev. Lett. 52 1-4

[13] Bouzouina A and De Bièvre S 1996 Equipartition of the eigenfunctions of quantized ergodic maps on the torus Comm. Math. Phys. 178 83-105

[14] Brody T A, Flores J, French J B, Mello P A, Pandey A and Wong S S M 1981 Random-matrix physics: spectrum and strength fluctuations Rev. Mod. Phys. 53 385-479

[15] Cornfeld I P, Fomin S V and Sinai Y G 1982 Ergodic Theory (Berlin: Springer-Verlag)

[16] Colin de Verdière Y 1985 Ergodicité et fonctions propres du Laplacien Comm. Math. Phys. 102 497-502

[17] Casati G and Prosen T 2000 The triangle map: a model of quantum chaos Phys. Rev. Lett. 85 4261-4264

[18] Casati G and Prosen T 1999 Quantum chaos in triangular billiards (unpublished).

[19] Casati G, Prosen T, Lan J and Li B 2004 Universal decay of classical Loschmidt echo of neutrally stable but mixing dynamics Preprint nlin.CD/0409018

[20] De Bièvre S 2001 Quantum chaos: a brief first visit Contemp. Math. 289, 161-218

[21] Degli Esposti M and Galatolo S 2004 Recurrence near given sets and the complexity of the Casati-Prosen map Chaos Solitons Fractals 23/4 1275-1284

[22] Degli Esposti M and Graffi S eds 2003 The Mathematical Aspects of Quantum Maps (volume 618 of Lecture Notes in Physics: Springer)

[23] Degli Esposti M and Graffi S 2003 Mathematical aspects of quantum maps. In [22] pp. 49-90

[24] De Bièvre S and Degli Esposti M 1998 Egorov theorems and equidistribution of eigenfunctions for quantized sawtooth and Baker map Ann. Inst. Henri Poincaré 69 1-30

[25] Drmola M and Tichy R F 1997 Sequences, Discrepancies and Applications (volume 1651 of Lecture Notes in Mathematics: Springer)

[26] Eckhardt B, Fishman S, Keating J, Agam O, Main J and Müller K 1995 Approach to ergodicity in quantum wave functions Phys. Rev. E 52 5893-5903

[27] Forrester P J and Witte N S 2000 Exact Wigner surmise type evaluation of the spacing distribution in the bulk of the scaled random matrix ensembles Lett. Math. Phys. 53 195-200 
[28] Furstenberg H (1961) Strict Ergodicity and Transformation of the Torus Amer. J. Math. 83 573-601

[29] Gérard P and Leichtnam É 1993 Ergodic properties of eigenfunctions for the Dirichlet problem Duke Math. J. $\mathbf{7 1} 559-607$

[30] Giraud O, Marklof J and O'Keefe S 2004 Intermediate statistics in quantum maps J. Phys. A 37 L303-L311

[31] Gorin T and Wiersig J 2003 Low rank perturbations and the spectral statistics of pseudointegrable billiards Phys. Rev. E 68065205 (R)

[32] Haake F 2000 Quantum signatures of chaos (Berlin: Springer-Verlag)

[33] Hannay J H and Berry M V 1980 Quantization of linear maps on the torus-Fresnel diffraction by a periodic grating Physica D $1267-290$

[34] Helffer B, Martinez A and Robert D 1987 Ergodicité et limite semi-classique Comm. Math. Phys. 109 313-326

[35] Hensley D and Su F E 2004 Random walks with badly approximable numbers; in Unusual Applications of Number Theory, volume 64 of DIMACS Ser. Discrete Math. Theoret. Comput. Sci. Amer. Math. Soc., pp. 95-102

[36] Keating J P 1991 The cat maps: quantum mechanics and classical motion Nonlinearity 4 309-341

[37] Keating J P, Mezzadri F and Monastra A G 2003 Nodal domain distributions for quantum maps J. Phys. A 36 L53-L59

[38] Mehta M L 1991 Random Matrices (Academic Press)

[39] Marklof J and O'Keefe S 2005 Weyl's law and quantum ergodicity for maps with divided phase space; Appendix by S Zelditch "Converse quantum ergodicity" Nonlinearity 18 277-304

[40] Marklof J and Rudnick Z 2000 Quantum unique ergodicity for parabolic maps Geom. Func. Anal. 10 1554-1578

[41] O'Keefe S Quantum eigenstates of linked twist maps Ph.D. Thesis, University of Bristol. In preparation.

[42] Robnik M and Berry M V 1986 False time-reversal violation and energy level statistics: the role of anti-unitary symmetry J. Phys. A 19 669-682

[43] Schnirelmann A I 1974 Ergodic properties of eigenfunctions Uspekhi Mat. Nauk 29 181-182

[44] Voros A 1979 Semi-classical ergodicity of quantum eigenstates in the Wigner representation. In Stochastic behaviour in classical and quantum Hamiltonian systems (Springer-Verlag) pp. 326-333.

[45] Zelditch S 1987 Uniform distribution of eigenfunctions on compact hyperbolic surfaces Duke Math. J. 55 919-941

[46] Zelditch S 1996 Quantum ergodicity of $C^{*}$ dynamical systems Comm. Math. Phys. 177 507-528

[47] Zelditch S 1997 Index and dynamics of quantized contact transformations Ann. Inst. Fourier (Grenoble) 47 305-363

[48] Zelditch S and Zworski M 1996 Ergodicity of eigenfunctions for ergodic billiards Comm. Math. Phys. 175 673-682 